\title{
THERMAL BIFURCATION
}

\section{AS A DRIVER OF}

\section{STELLAR SURFACE INHOMOGENEITIES}

\author{
It's Probably Not Too Important, But Read On!
}

\author{
T.R. AYRES \\ Center for Astrophysics and Space Astronomy \\ University of Colorado, Boulder, CO, USA
}

\begin{abstract}
Cool gas in the heart of the hot solar chromosphere betrays itself through anomalously strong absorptions of $4.7 \mu \mathrm{m}$ CO lines at the extreme limb, and off-limb emissions. The origin of the "cool clouds" is thought to be a thermal instability driven by the $\mathrm{CO}$ itself. Questions concerning the pervasiveness and detailed structure of the "thermally-bifurcated" zones are being addressed through stigmatic spectroscopy of the solar CO bands with the NSO McMath-Pierce telescope. Here, I report recent progress, and implications for surface thermal inhomogeneities on other stars.
\end{abstract}

\section{Introduction}

It has now been quite a long time since the first high-quality studies of the infrared $\Delta v=1$ (fundamental) bands of carbon monoxide in the Sun (e.g., Noyes \& Hall 1972a,b) and the stars (Heasley et al. 1978). In both cases, anomalously strong absorption cores in the deepest of the $4.7 \mu \mathrm{m} \mathrm{CO}$ lines signaled the presence of cool material high in the stellar photosphere, whose origins could not easily be understood. Subsequent work with the large Fourier transform interferometer (Brault 1979) on the McMath solar telescope ${ }^{1}$ at Kitt Peak suggested that the cool material was present even within the chromosphere itself (Ayres \& Testerman 1981). It was a surprising conclusion given that the previous two decades of solar physics largely had been devoted to the origin of line-core emission reversals and the chromospheric temperature inversion (Athay 1976).

${ }^{1}$ Since rededicated the McMath-Pierce, in honor of Keith Pierce. 
At the time-nearly fifteen years ago-I proposed that the "cool clouds" above the photosphere (with temperatures as low as $\approx 3600 \mathrm{~K}$ ) could be understood as a consequence of strong $\mathrm{CO}$ surface cooling in areas where the local mechanical heating was weak (Ayres 1981). That interpretation drew considerable criticism over the intervening years, but also received cautious support in the NLTE-blanketed energy-balance models of Anderson (1989) and Anderson \& Athay (1989), and in other studies (see, e.g., Ayres \& Rabin 1995 [AR], and references therein).

More recently, the "cold heart" 2 of the solar chromosphere has revealed itself directly through off-limb emissions of the CO bands (Solanki, Livingston, \& Ayres 1994 [SLA]). Further, long-slit imaging with the McMathPierce Main spectrograph and an IR camera has mapped the solar surface in temperature and velocity in the light of high-altitude $\mathrm{CO}$ absorptions (Uitenbroek, Noyes, \& Rabin 1994 [UNR]; Uitenbroek \& Noyes 1994 [UN]; AR). The off-limb emissions combined with the normal-incidence surface maps strongly constrain the amount of cool material in the low chromosphere, and how it is organized spatially (e.g., AR; and below).

Most indications at present point to a pervasive occupation of the low chromosphere by cool gas, up to an altitude of $h \approx 1000 \mathrm{~km}$ (above $\tau_{500 \mathrm{~nm}}=$ 1). Previously, it had been supposed that the photosphere extended only up to about $500 \mathrm{~km}$ (e.g., Maltby et al. 1986 [MACKKL]: model VAL $\mathrm{C}^{\prime}$ ). In reality, the true upper boundary appears to coincide with the higher-lying "canopy" (of merged magnetic flux ropes from the supergranulation network); although, of course, some small-scale hot chromospheric structures penetrate down through the cool zone (the "COmosphere" ${ }^{3}$ ), giving rise to a sparse bright-point pattern in the $\mathrm{CO}$ thermal images, and in other diagnostics like the optical CN $388 \mathrm{~nm}$ and CH $430 \mathrm{~nm}$ bands (Sheeley 1969; Berger et al. 1995).

The current study of the $\mathrm{CO}$ bands has benefitted greatly on the observational side from access to imaging spectroscopy, made possible by the new generation of IR sensors. On the theoretical side, the development of multidimensional radiative transfer simulations (which thankfully can be done in LTE for the CO $\Delta v=1$ bands) has allowed the numerical treatment of problems-like the off-limb CO emissions-with a significant amount of geometrical complexity. While the physical mechanism of thermal bifurcation is relatively weak in the Sun compared with, for example, the red giants (see AR); we observers still are strongly drawn to the solar example, where the possibility exists to directly visualize the surface features and probe their structure from a variety of viewing angles.

\footnotetext{
${ }^{2}$ Sami S. prefers "heart of darkness."

${ }^{3}$ Not my idea: G. Wiedemann's.
} 
Here, I will report recent solar results, and speculate on the role that $\mathrm{CO}$-mediated "cooling catastrophes" might play in inspiring strong thermal contrasts in the atmospheres of other stars.

\section{Long-Slit Imaging Spectroscopy of the Solar CO bands}

About two years ago, the National Solar Observatory put into operation a long-slit stigmatic imaging system for thermal infrared work at the McMathPierce. The current setup utilizes a $256 \times 256$ IR camera, and the telescope control system of the Near Infrared Magnetograph (Rabin 1994). (Incidentally, the $4.7 \mu \mathrm{m} \mathrm{CO}$ lines have no intrinsic magnetic sensitivity, so the NIM is operated in an unpolarized mode.) A $2.5 \mathrm{~cm}^{-1}$ interval of the spectrum can be recorded on the detector. The theoretical resolving power of the large IR grating in 2nd-order is $1 \times 10^{5}$, but in practice the apparent resolution is considerably lower $\left(\approx 6 \times 10^{4}\right)$, probably due to high levels of scattered light. ${ }^{4}$ Figure 1 illustrates a cleaned NIM frame, and a comparison of a trace of the central portion of the spectrogram with quiet-Sun scans from the high-resolution NSO/FTS (Livingston \& Wallace 1991) and the Shuttle-borne ATMOS/FTS (Farmer \& Norton 1989).

\subsection{TIME-RESOLVED THERMAL AND VELOCITY MAPS}

The NIM not only manages the camera operations, but also can step the solar image across the slit to build up spectral maps of the surface. The variations of line core depth and Doppler shift in each frame can be translated into 1-D distributions of temperature and velocity fluctuations. Stepping the slit then yields a 2-D map. Repeating a multi-step swath can produce a "movie." In the most recent run by myself and D. Rabin (1995 May), we used a 0 ." $4 \times 50 "$ slit ( $0 . " 2$ pixels along the slit). The integration time was only $0.14-0.3 \mathrm{sec}$, although unavoidable overheads raised the typical cadence to one frame per $3 \mathrm{sec}$. Figure 2 depicts a movie sequence of thermal and velocity maps (each covering a field of 16 " $\times 40$ "), taken in a quiet region at disk center on the morning of 1995 May 4, under relatively good observing conditions (aside from occasional clouds). The interval between frames was fixed at $2.5 \mathrm{~min}$, in order to catch essentially opposite phases of the 5 min $p$-mode oscillations.

Previous low-spatial resolution CO time series (Ayres \& Brault 1990) had indicated that the global oscillations are the major cause of the intensity and line-shift variations in the CO spectra, and subsequent NIM/CO maps (UNR; UN; AR) confirmed their central role. One sees here further

\footnotetext{
${ }^{4}$ Easily compensated in a single-channel double-pass scanning system of the type originally used, but more difficult to treat in the present 2-D imaging configuration.
} 
$\leftarrow$ dispersion $\rightarrow$

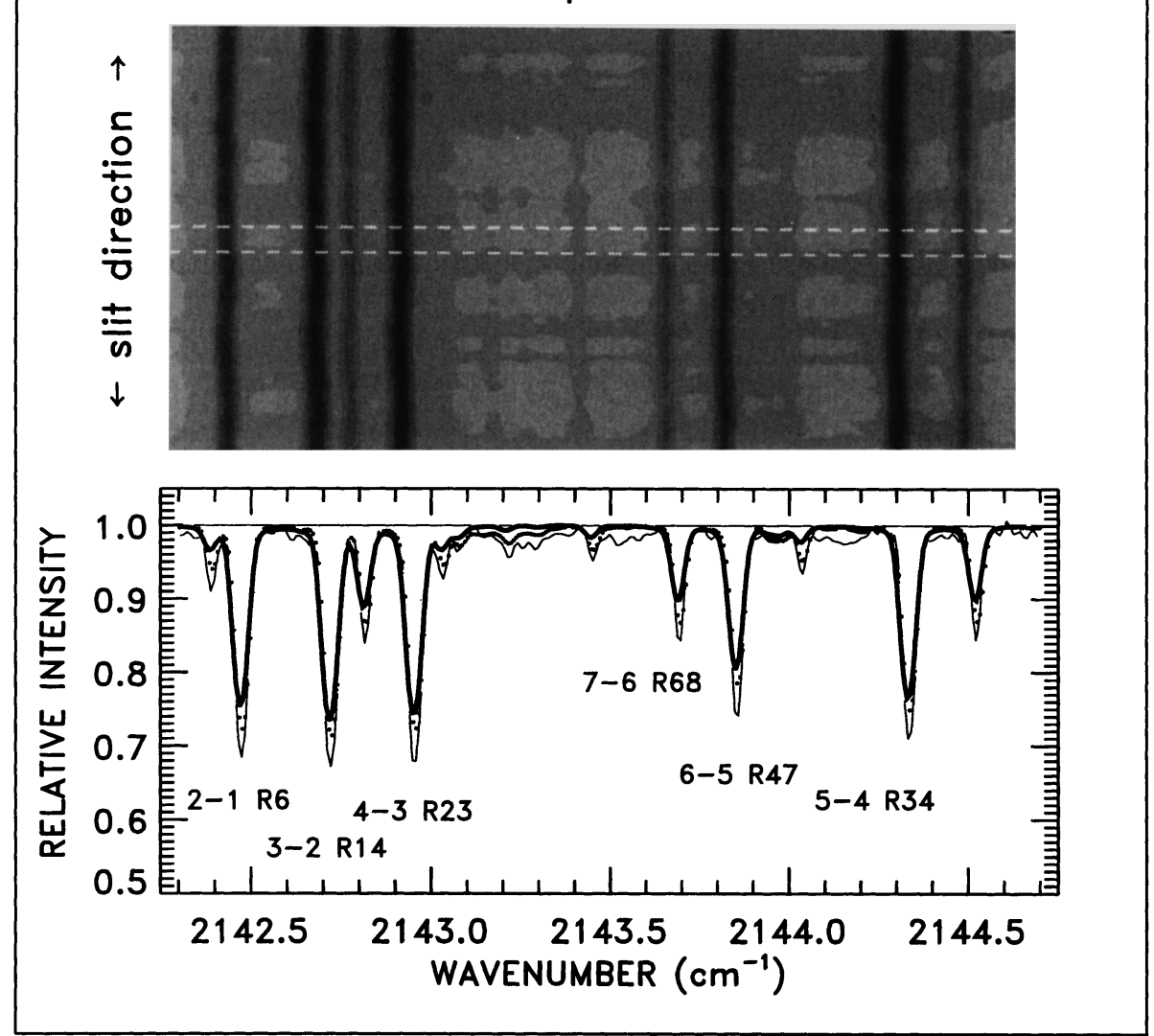

Figure 1. Upper: cleaned camera frame. Lower: trace of NIM spectrum (heavy curve) compared with NSO/FTS (light: $\omega / \Delta \omega \approx 2 \times 10^{5}$ ) and ATMOS (dots: $9 \times 10^{4}$ ).

affirmation of that picture. The velocity map is dominated by distinct timevarying patches of coherent motion, phased according to the $p$-modes. Yet, the maximum velocities $\left(\approx 1000 \mathrm{~m} \mathrm{~s}^{-1}\right)$ are only a fraction of the local sound speed. Similarly, the temperature fluctuations are patchy and timevariable, yet the maximum excursions are relatively modest. Indeed, if one were to filter out the $p$-mode signatures in temperature and velocity, the remaining underlying thermal structure would be uniform and static.

Thus, there is little direct imaging evidence for strong thermal inhomogeneities in the mid-photosphere, an important constraint on models that 


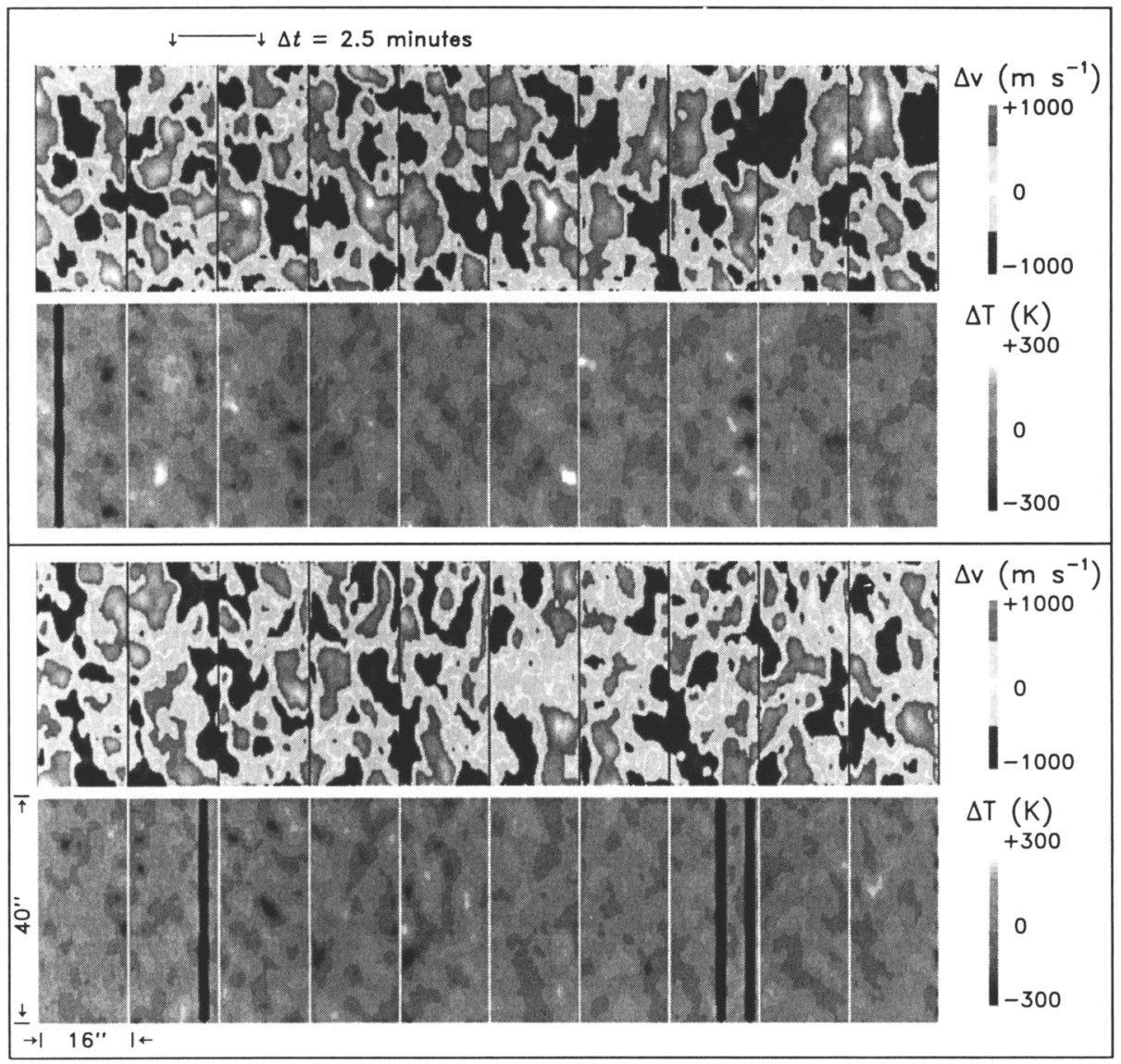

Figure 2. Thermal and velocity maps from $50 \mathrm{~min}$ NIM/CO time series. Within each panel, the upper frames are $\Delta v$ 's and the lower frames are $\Delta T$ 's. The color tables emphasize the extremes. Time runs from left to right, from top to bottom. The dark vertical stripes represent cloud-affected frames.

would explain the anomalous extreme-limb behavior of the CO $4.7 \mu \mathrm{m}$ lines through "shadowing" by small-scale sparsely-distributed ultra-cold structures in the outer photosphere (see AR).

Incidentally, the imaging sequence shown above was part of a coordinated two-site campaign to obtain multi-spectral time series of solar surface structure. Our colleague L. November used the Tower telescope at Sac Peak to record simultaneous, cospatial images in a wide variety of optical diagnostics, including $\mathrm{CN}, \mathrm{Ca}$ II, the $400 \mathrm{~nm}$ continuum, magnetically-sensitive lines, and so forth. The complete data set still is being analyzed and will be described elsewhere. 


\subsection{OFF-LIMB CO EMISSIONS}

The remarkable off-limb emissions of $\mathrm{CO}$ originally were discovered using the upgraded Main spectrograph of the McMath-Pierce, but with a conventional single-channel scanning detector system (SLA). Subsequently, the off-limb emissions have been recorded using the NIM/CO long-slit imager; in the quiet-Sun (UNR; AR) and during the 1994 May partial eclipse (Clark et al. 1995). The CO is in emission above the limb because the cores of the strongest lines still are quite opaque along grazing rays for which the infrared continuum has become thin (the continuum edge corresponds to an altitude of about $350 \mathrm{~km}$ above $\left.\tau_{500 \mathrm{~nm}}=1\right)$. One therefore still sees the blackbody radiation from the line core, even when the surrounding thermal emission from the (now optically thin) continuum has faded away.

The off-limb emissions are a crucial diagnostic for the radial extent of the COmosphere, and an indirect indicator for the filling factor of the cool material. Figure 3 depicts two realizations of inhomogeneous two-component models of the solar outer atmosphere, and the corresponding 2-D (LTE) numerical simulations of the extreme limb-and off-limb-CO spectra (see $\mathrm{AR}$ for details). The hot component is taken to be the VAL $\mathrm{C}^{\prime}$ reference model of MACKKL. The cool COmospheric extension(s) have thermal profiles designed to match the observed center-to-limb behavior-and off-limb emissions-of the CO lines. In the case of "Scenario Zero", there is a $20 \%$ filling factor of the hot component, arranged in $1 \mathrm{Mm}\left(=10^{3} \mathrm{~km} \approx 1 . " 4\right)$ wide structures on $5 \mathrm{Mm}$ centers. In the cool component, the chromospheric temperature inversion is about $500 \mathrm{~km}$ higher than that of the reference model, and the minimum temperature is $1500 \mathrm{~K}$ cooler. The elevated inversion coincides with the putative "canopy" of merged network magnetic fields (SLA), and also is approximately the acoustic shock formation height indicated in recent 1-D radiation/hydro simulations (Carlsson \& Stein 1994).

The 2-D simulations for Scenario Zero show that the cool component strongly "shadows" the hotter gas at the extreme limb, further suppressing its already small filling factor. The shadowing is of little consequence, however, because the cool component already dominates the $\mathrm{CO}$ formation at all viewing angles.

Figure 4 compares the predictions of Scenario Zero with the observed off-limb extensions of the CO lines in the key $2143 \mathrm{~cm}^{-1}$ interval (a region containing weak and strong CO lines, and relatively free of telluric contamination). The observations were seeing-selected from several long sequences at the South heliocentric limb, taken on 1994 April 29. The off-limb emissions show little variation with position along the limb. The strong $\mathrm{CO}$ lines (e.g., 2-1 R6) extend about 0." 6 (nominally $450 \mathrm{~km}$ ) beyond the continuum edge (at the $40 \%$ intensity level, where $100 \%$ refers to a position 


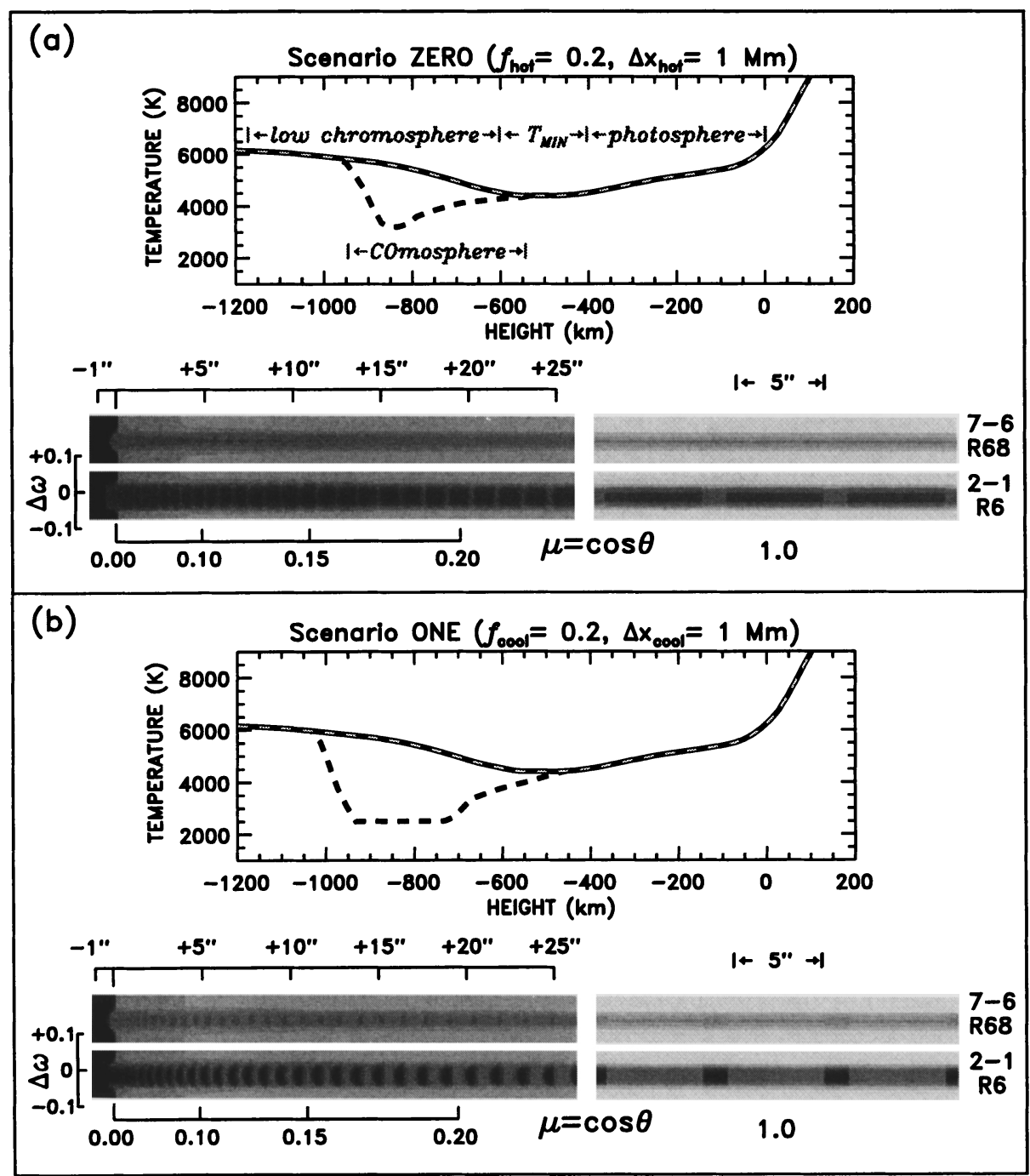

Figure 3. Inhomogeneous two-component models and 2-D spectral simulations (limb is to left; disk center to right). In each panel, upper spectrum is for weak 7-6 R68 line, and lower spectrum is for strong $2-1 \mathrm{R} 6$.

at $\mu \approx 0.15$ on the disk), while the weaker lines (e.g., 7-6 R68) extend to about $0 . " 3$. The simulated 2-D spatially-resolved spectrum was degraded to 1 " resolution along the slit (the best expected with the McMath-Pierce, in view of the 0 " 8 diffraction limit at $4.7 \mu \mathrm{m}$ ), and convolved spectrally with a simulated instrumental profile (sharp Gaussian core, but with a powerlaw scattering "halo"). The latter, unfortunately, still is poorly determined, 


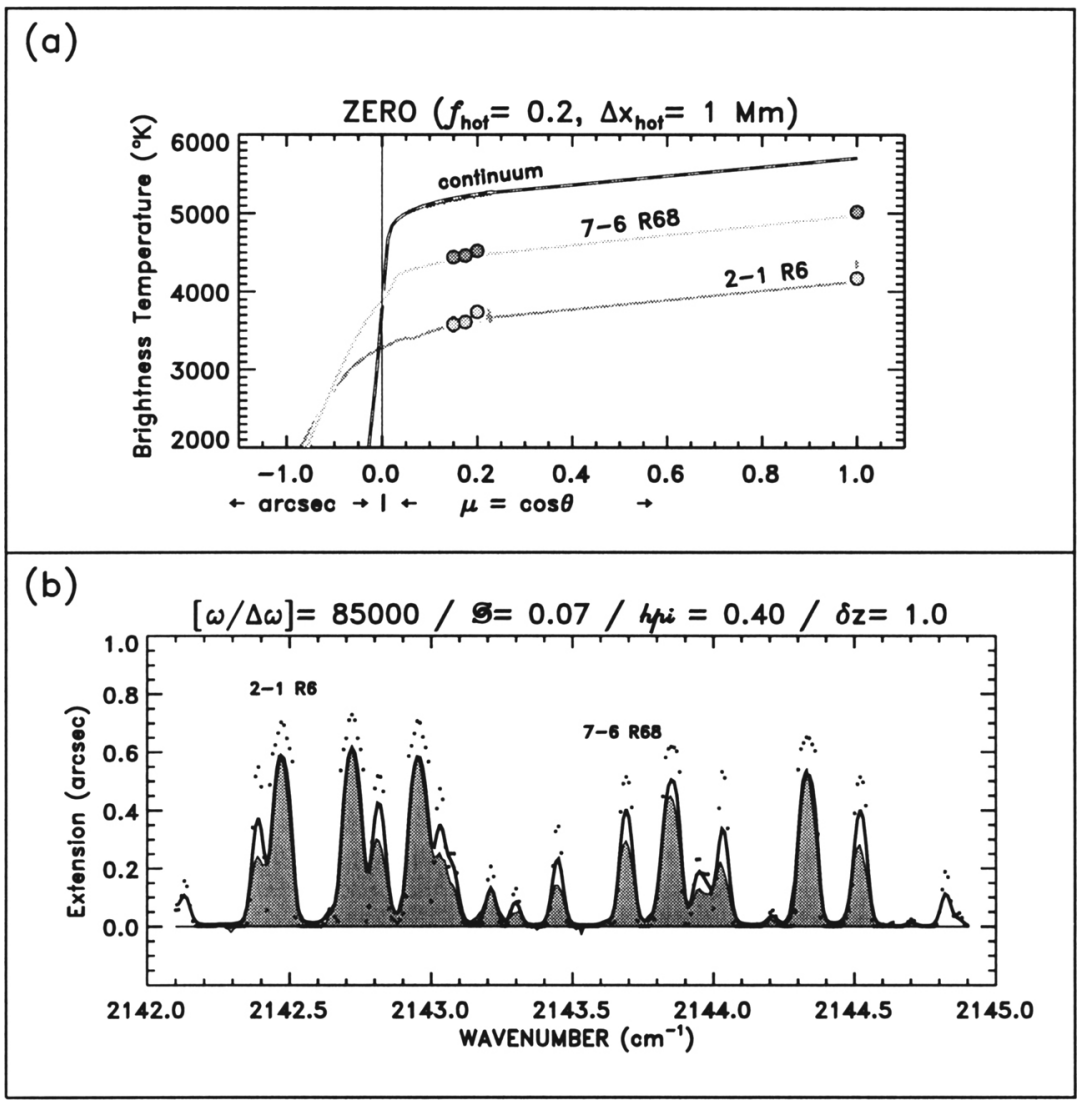

Figure 4. (a) Predicted center-to-limb behavior for Scenario Zero (light curves are for CO; dashed, for the continuum; large dots represent NSO/FTS measurements). (b) Predicted off-limb CO extensions for Scenario Zero (heavy solid curve), compared with observations (shaded). The small dots represent the original calculations (i.e., before degradation by the instrumental profile and seeing).

pending direct measurements (which heretofore have not been practical). The simulated spectrum fits the strong lines well, but overestimates the weaker lines. Although one might be tempted to gloss over the poor fit of the weaker lines, it potentially is a key diagnostic for the true loading of molecular material along the sightline through the low chromosphere. 
Figure 5 depicts the 2-D predictions of the opposite scenario (see Fig. 3): $80 \%$ hot component, with a $20 \%$ cool component in $1 \mathrm{Mm}$ wide structures on $5 \mathrm{Mm}$ centers. Scenario One more closely matches the weaker lines, butat least at the NIM/CO resolution(s) - fails to reproduce the stronger lines. More damaging, the majority-hot-component scenario predicts a $20 \%$ population of ultracold $(\Delta T \approx-1000 \mathrm{~K})$ dark points in a disk-center thermal map (Fig. 3), contrary to the existing NIM/CO observations (e.g., of UNR and AR). Nevertheless, the lack of agreement of the first scenario (majority cool component) with the weak-line off-limb extensions should be investigated further. Indeed, Avrett (1995) recently has proposed a $60 \%$ cool + $40 \%$ hot scenario based on a joint analysis of the CO $4.7 \mu \mathrm{m}$ lines (from the ATMOS/FTS), and far-UV continuum intensities (an excellent tracer of the hot component). Alternatively-as strongly advocated by Rob Rutten at the present symposium-1-D time-dependent wave models like the simulations of Carlsson \& Stein (1994) might resolve the bifurcation dilemma in a more natural way than the 2-D, but static, spatially-inhomogeneous planforms of the present work.

\section{Thermal-Bifurcation of Sunspots}

The lingering question of the detailed thermal structure of the cool COmospheric component led myself and D. Rabin to search for evidence of off-limb emissions of water vapor in the quiet Sun. Water (or, more precisely, steam) was identified in sunspot umbrae nearly two decades ago, but has not yet been found in the quiet Sun (Wallace et al. 1995). We reasoned that if the temperatures in the COmosphere dropped as low as the $2500 \mathrm{~K}$ indicated in the NLTE-blanketed RE simulations of Anderson (1989), then an appreciable amount of $\mathrm{HOH}$ might form and be detectable off-limb. Thus, Doug and I conducted a thorough search of a key interval in the $2453 \mathrm{~cm}^{-1}$ region, known to harbor strong water (and hydroxyl) absorptions in sunspot spectra. We obtained a thousand or so frames at the North limb (under not particularly good conditions), and seeing-selected the best $\approx 10$. We carefully registered, and co-added the selected frames, and evaluated the monochromatic extensions in the same way as for the $\mathrm{CO}$ lines. Several weak "blips" were seen close to the correct wavenumbers of the sunspot $\mathrm{HOH}$ and $\mathrm{OH}$ lines. With success glinting in our eyes, we then set about calculating off-limb extensions to compare with the spectral distribution we had recorded.

Unfortunately, the specific $\mathrm{HOH}$ and $\mathrm{OH}$ features did not have readily available energy levels or oscillator strengths. Our only recourse was to empirically estimate the absorption strengths by reference to the apparent depths of the features in the NSO/FTS sunspot spectrum (Wallace \& 


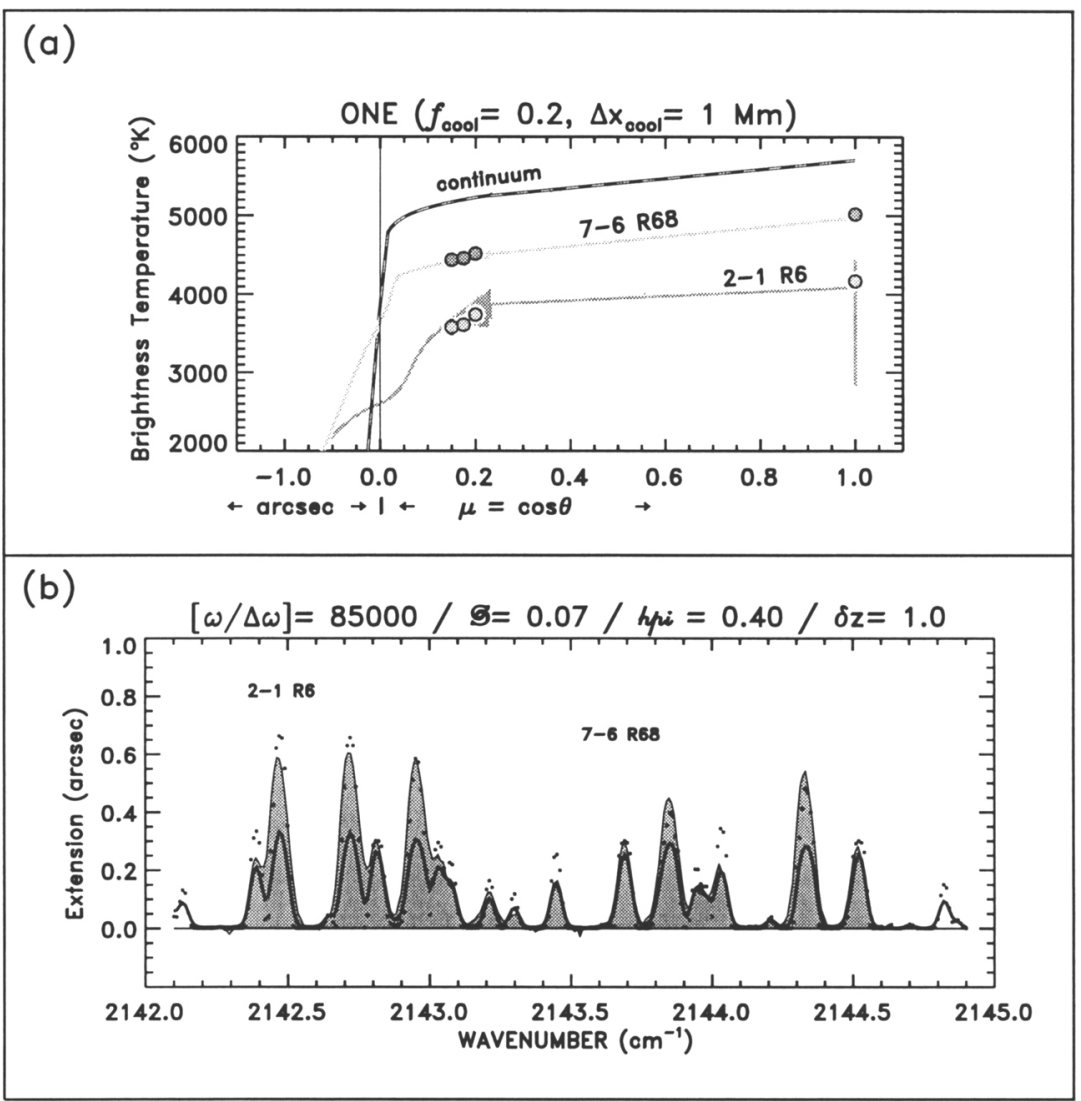

Figure 5. Same as Fig. 4, for Scenario One.

Livingston 1992). That comparison in turn required the specification of an umbral thermal profile. The best available sunspot reference model is that of MACKKL (also the source of the quiet-Sun reference model).

As a preliminary step to validate the reference thermal profile, we simulated the $\mathrm{CO} 4.7 \mu \mathrm{m}$ lines using the MACKKL model. To our surprise, it predicted prominent emission cores in the strongest of the $\mathrm{CO}$ absorptions, contrary to the observations. Exactly that situation faced Heasley et al. (1978) in their pioneering stellar-FTS measurements of the red gi- 
ant Arcturus: the empirical CO lines displayed cold cores, whereas the best Arcturan chromospheric models (based on the ultraviolet Ca II and Mg II lines) predicted strong emission reversals. A thermally-bifurcated atmosphere-with sparsely-distributed network bright points-is not difficult to imagine in ancient low-mass giants like Arcturus, which inhabit the depths of the coronal graveyard (Ayres, Fleming, \& Schmitt 1991). But, finding a comparable bifurcation in the heart of a solar active region was frankly unexpected. Figure 6 illustrates the dichotomy. We found it necessary to extend the MACKKL photosphere to higher altitudes and cooler temperatures in order to match the residual intensity spectra of the CO bands; in much the same way that Ayres \& Testerman (1981) were forced to modify solar chromospheric reference models to accommodate CO.

The revised sunspot reference model bolstered our confidence in our predictions of the $\mathrm{HOH}$ and $\mathrm{OH}$ line strengths. We determined the $\mathrm{HOH}$ concentration based on the key gas-phase reaction between $\mathrm{OH}$ and $\mathrm{H}_{2}$, and equilibrium concentrations of the two parent species. Ironically, our simulations indicated that the $\mathrm{HOH}$ (and $\mathrm{OH}$ ) lines arise in the deeper layers of the umbral photosphere (in hindsight, because of the strong density dependence of the $\mathrm{HOH}$ formation). Thus our modifications to the outer umbral photosphere had very little impact on the outcome.

Armed with the empirical line strengths, and already a catchy name for the water zone (the "hydrosphere"), we conducted a spectral simulation of the off-limb emissions of $\mathrm{HOH}$ and $\mathrm{OH}$. Much to our disappointment, even rather extreme COmospheric models predicted entirely negligible off-limb extensions for the water lines. We did successfully match the $\mathrm{OH}$ features, but hydroxyl already was known in the quiet Sun, and its puny off-limb extension is of little or no diagnostic value. In hindsight, a detailed examination of the high S/N telluric-free ATMOS atlas revealed weak absorptions slightly displaced from the sunspot water lines, but corresponding exactly in wavenumber to the features whose off-limb extensions we initially had attributed to water. The identity of the features is unknown, but they apparently are not water.

Thus, the hydrosphere devolved immediately into the HOHo-sphere. In retrospect, the amount of possible water at high altitudes-even with gas temperatures uniformly and pervasively below $3000 \mathrm{~K}$-is too slight to make a readily detectable signature in the off-limb frames. Nevertheless, the sleuthing led us to a more important recognition: even sunspotsmaelstroms of magnetic activity - are not immune to the high-altitude thermal differentiation processes that apparently operate in quiet regions. 


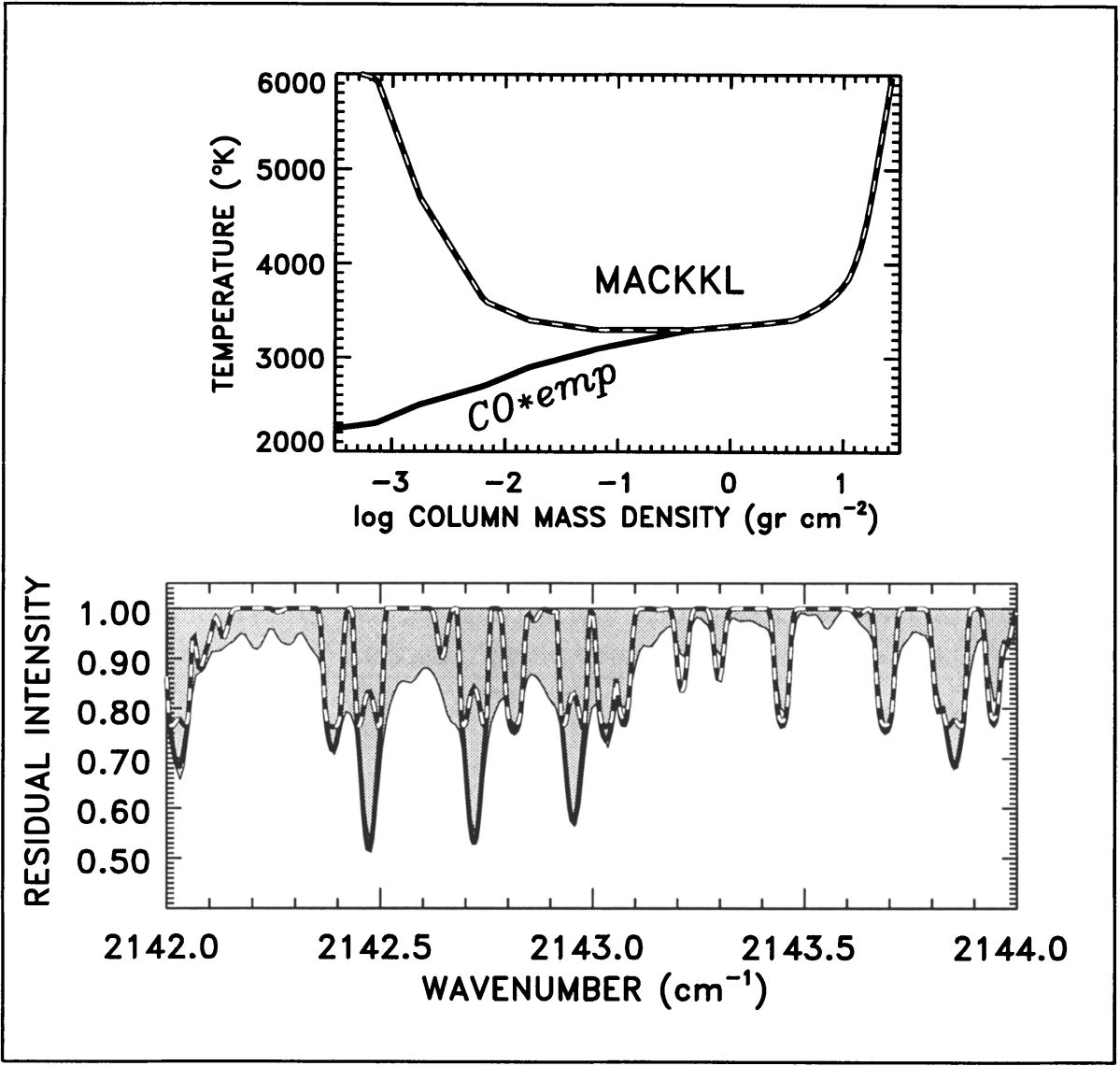

Figure 6. Comparison of predicted sunspot spectra with NSO/FTS umbral reference atlas (shaded). Dashed curve is for the MACKKL reference spot model; heavy solid curve is for the empirical modification (to fit the CO bands).

\section{A Driver for Stellar Surface Structure?}

Thermal bifurcation, per se, largely is a high-altitude phenomenon in the Sun and in the stars. Thus it should have little, or no, influence on the thermal structure of the deeply-seated photospheric layers, that are sensed most directly by photometric and Doppler-imaging surface-reconstruction techniques. Nevertheless, the historical diagnostic dilemma-cold cores in strong $\mathrm{CO}$ lines vs. emission reversals in $\mathrm{Ca}$ II $\mathrm{H}$ and $\mathrm{K}$-pervades not only the quiet Sun, but active regions as well. Thus, our understanding of the intrinstic thermal profile(s) of the solar chromosphere-extending from the quiet-Sun to sunspots - cannot be complete until the dilemma is resolved. Deriving accurate thermal profiles is at the heart of efforts to probe 
the plasma energy balance and heating mechanisms (Vernazza, Avrett, \& Loeser 1981). If we don't understand so simple a star as the Sun, it would be naively foolish to attempt to comprehend the truly complex beasts that inhabit the zoo of stellar magnetic hyperactivity.

Acknowledgments. I thank D. Rabin for his help; D. Jaksha and C. Plymate provided technical assistance. This work was supported by grant AST9218063 from the National Science Foundation. The observations were obtained at the National Solar Observatory, NOAO, operatea for the NSF by the Association of Universities for Research in Astronomy, Inc.

\section{References}

Anderson, L. S. 1989, ApJ, 339, 558

Anderson, L. S., \& Athay, R. G. 1989, ApJ, 346, 1010

Athay, R. G. 1976, The Solar Chromosphere and Corona: Quiet Sun, (Dordrecht: D. Reidel)

Avrett, E. H. 1995, in Infrared Tools for Solar Astrophysics: What's Next?, eds. J. Kuhn \& M. Penn (Singapore: World Scientific), 303

Ayres, T. R. 1981, ApJ, 244, 1064

Ayres, T. R., \& Brault, J. W. 1990, ApJ, 363, 705

Ayres, T. R., Fleming, T. A., \& Schmitt, J. H. M. M. 1991, ApJ, 376, L45

Ayres, T. R., \& Rabin, D. 1995, ApJ, (submitted) (AR)

Ayres, T. R., \& Testerman, L. 1981, ApJ, 245, 1124

Berger, T., Shine, R., Tarbell, T., Title, A., \& Scharmer, G. 1995, BAAS, 26, 1465

Brault, J. 1979, Osservazioni e memorie dell' Osservatorio Astrofis. di Arcetri, 106, 33

Carlsson, M., \& Stein, R. F. 1994, in Chromospheric Dynamics, ed. M. Carlsson (Oslo: Institute of Theoretical Astrophysics), 79

Clark, T. A., Lindsey, C., Rabin, D. M., \& Livingston, W. C. 1995, in Infrared Tools for Solar Astrophysics: What's Next?, eds. J. Kuhn \& M. Penn (Singapore: World Scientific), 133

Farmer, C. B., \& Norton, R. H. 1989, A High-Resolution Atlas of the Infrared Spectrum of the Sun and Earth Atmosphere from Space, Vol. 1: The Sun, NASA RP-1224

Heasley, J. N., Ridgway, S. T., Carbon, D. F., Milkey, R. W., \& Hall, D. N. B. 1978, ApJ, 219, 790

Livingston, W., \& Wallace, L. 1991, An Atlas of the Solar Spectrum in the Infrared from 1850 to $9000 \mathrm{~cm}^{-1}$ (1.1 to $5.4 \mathrm{\mu m}$ ), (Tucson: NOAO) NSO Tech. Rept. No. 91-001

Maltby, P., Avrett, E. H., Carlsson, M., Kjeldseth-Moe, O., Kurucz, R. L., \& Loeser, R. 1986, ApJ, 306, 284 (MACKKL)

Noyes, R. W., \& Hall, D. N. B. 1972a, BAAS, 4, $389(\mathrm{NH})$

$$
\text { 1972b, ApJ, 176, L89 (NH) }
$$

Rabin, D. M. 1994, in IAU Symp. 154, Infrared Solar Physics, ed. D. M. Rabin, J. T. Jefferies, \& C. Lindsey (Dordrecht: Kluwer), 449

Sheeley, N. R. 1969, Solar Phys., 9, 347

Solanki, S. K., Livingston, W., \& Ayres, T. 1994, Science, 263, 64 (SLA)

Uitenbroek, H., \& Noyes, R. W. 1994, in Chromospheric Dynamics, ed. M. Carlsson (Oslo: Institute of Theoretical Astrophysics), 129 (UN)

Uitenbroek, H., Noyes, R. W., \& Rabin, D. 1994, ApJ, 432, L67 (UNR)

Vernazza, J. E., Avrett, E. H., and Loeser, R. 1981, ApJS, 45, 635

Wallace, L., et al. 1995, Science, 268, 1155

Wallace, L., \& Livingston, W. 1992, An Atlas of a Dark Sunspot Umbral Spectrum from 1970 to $8640 \mathrm{~cm}^{-1}$ (1.16 to $\left.5.1 \mu \mathrm{m}\right)$, (Tucson: NOAO) NSO Tech. Rpt. No. 92-001 


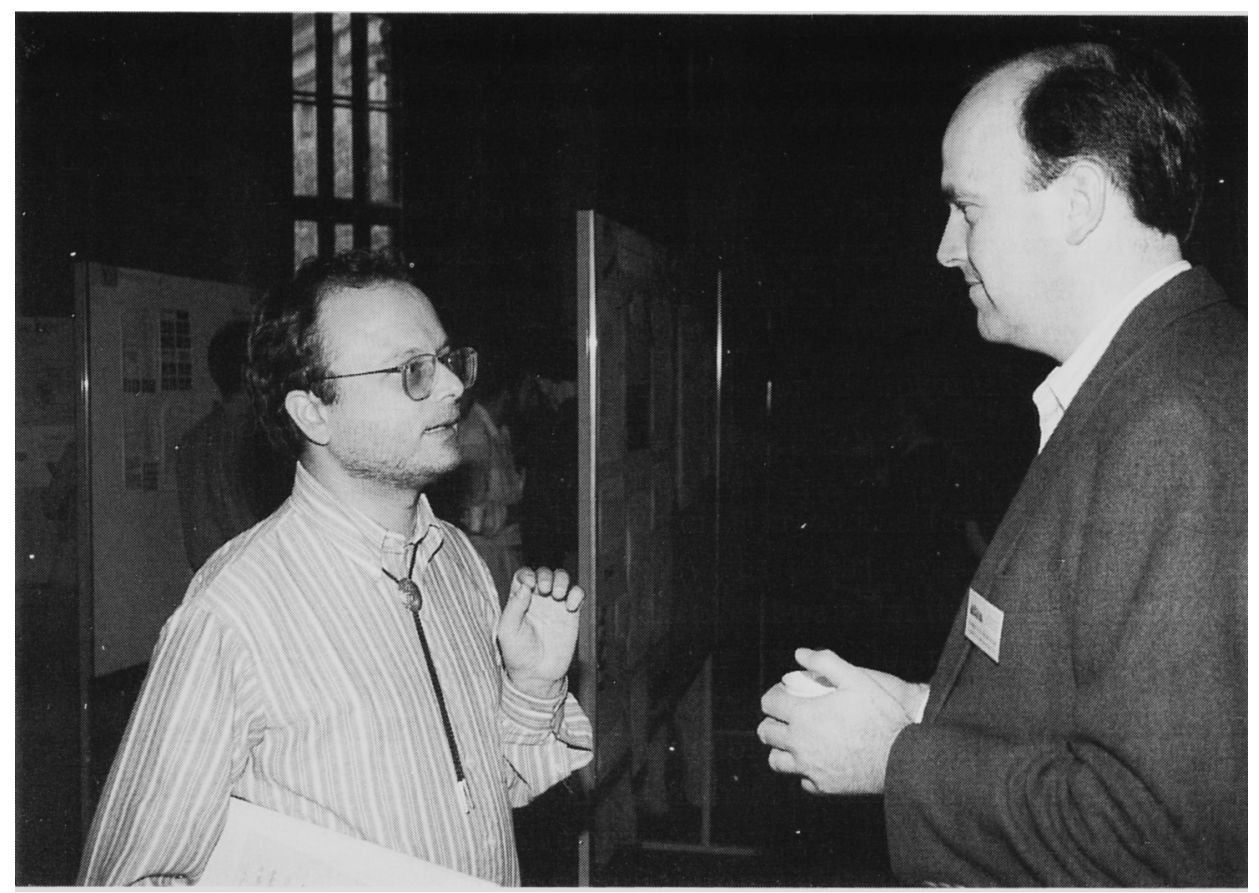

Manfred Cuntz tries to convince Karel Schrijver that the Sun is a normal star...

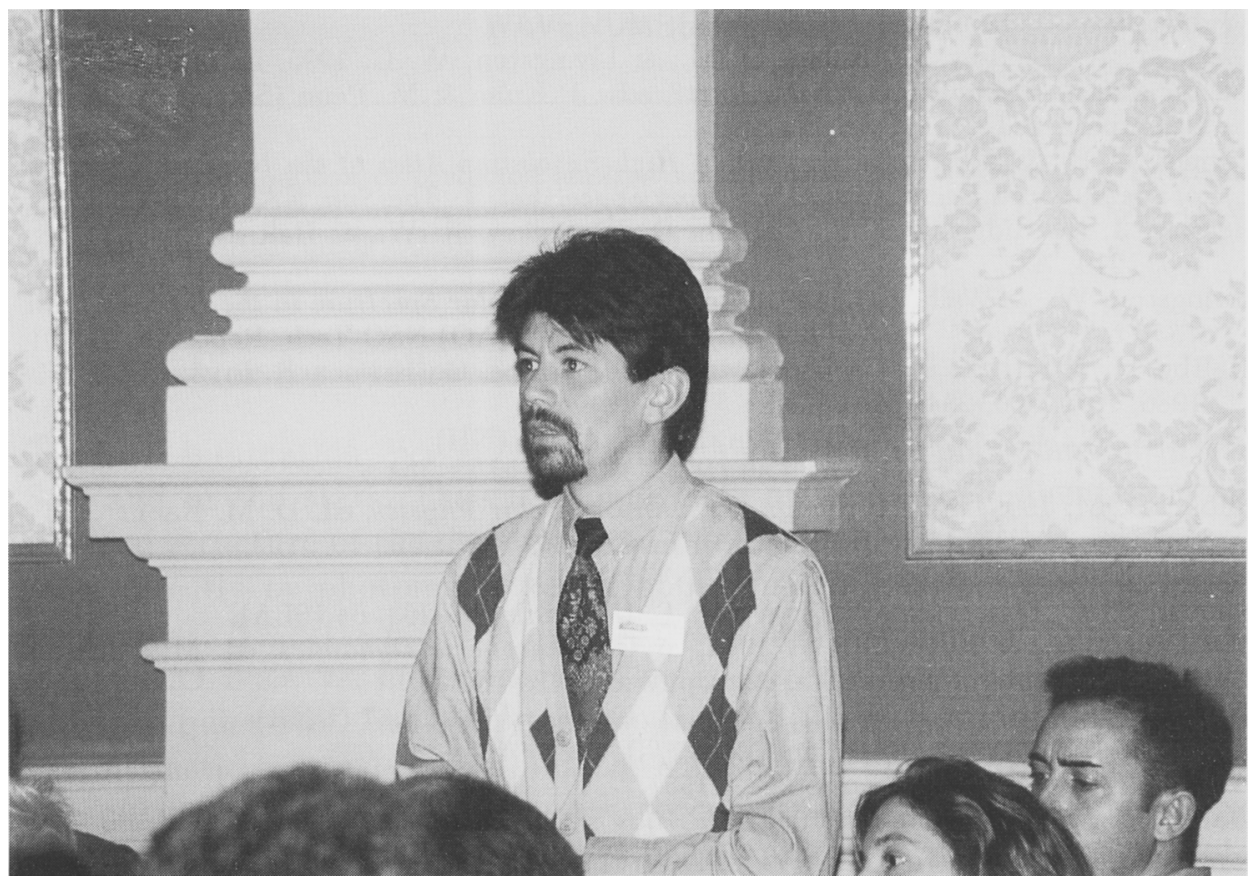

Bernard Foing: "I have five more questions and six comments ..." 